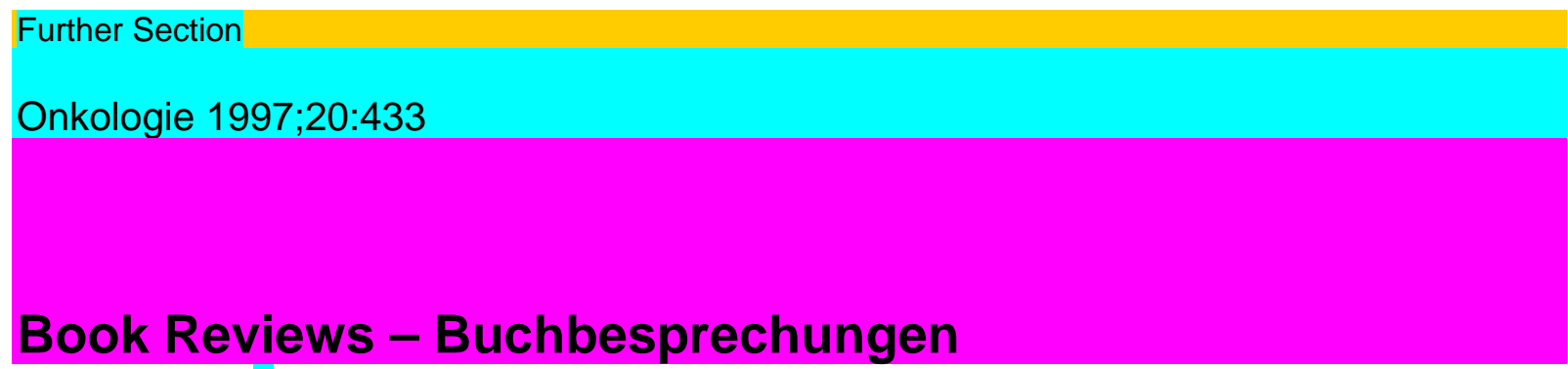

\title{
Solide Tumoren
}

Therapieleitlinien der internistischen Onkologie

Herausgeber: Deutsche Gesellschaft fur Hämatologie und

Onkologie (DGHO)

W. Zuckschwert-Verlag, München, 1. Auflage 1996

240 S., DM $48-$

ISBN 3-88603-560-3

Unter der redaktionellen Leitung von E. Heidemann aus Stuttgart zeichnen 13 weitere Autoren für die erste Auflage dieses Buches über Therapieleitlinien solider Tumoren ver-antwortlich. Wie in den Vorbemerkungen des 220 Seiten umfassenden Kompendiums ausgeführt, ist es das Ziel knapper alls dies in Behandlungsprotokollen möglich ist -, den jeweils aktuellen «Standard» für einzelne Tumorenti-täten darzustellen.

Nach zwei kurzen Kapiteln über qualitätssichernde Maßnah-men und rationelle Diagnostik in der Onkologie werden in der Folge sämtliche Entitäten solider Tumoren des Erwach-senenalters abgehandelt. In durchaus unterschiedlicher Aus-führlichkeit wird bei den meisten Tumorentitäten auf die Krankheitscharakteristik, die Stadieneinteilung, Prognose-faktoren sowie eventuelle adjuvante, kurative bzw. palliative Therapiemaßnahmen eingegangen. So werden beispielsweise das Kolon- und Mammakarzinom auf zwei bzw. vier Seiten des kleinformatigen Buches abgehandelt, während den Knochen- und Weichteiltumoren mehr als 30 Seiten gewidmet sind. Bei einzelnen Tumorentitäten wird auf Nachsorge-maßnahmen hingewiesen. Auf Literaturhinweise wird durch-wegs verzichtet.

Im Anschluß an ein kurzes Kapitel über metastasierende Tumoren mit unklarer primärer Lokalisation folgt ein sehr gut gegliedertes, praxisrelevante Leitlinien enthaltendes, gut gelungenes Kapitel über chronische Schmerzen des Tumor-kranken. Es folgen zwei Kapitel zu den Themen «lnfektionen bei Neutropenie» sowie «lndikationen zum Einsatz von Wachstumsfaktoren». Den Abschluß bildet eine Stellung-nahme zu sogenannten alternativen Heilmethoden, in der stichwortartig Merkmale derartig ungeprüfter Therapieverfahren sowie die Zielsetzungen und Gründe für die An-spruchnahme solcher Methoden aufgelistet werden.

Nachdem mögliche Ursachen für die Ablehnung wissenschaftlicher Therapieverfahren durch Patienten angeführt werden, endet das Kapitel mit Hinweisen für den behandelnden Arzt, wie er sich in einer solchen Situation im Umgang mit seinen Patienten verhalten sollte.

Zusammenfassend kann das handlich und übersichtlich gestal-tete Kompendium als möglicher Zusatz zu ausführlichen Stan-dardwerken angesehen werden, dies zur raschen und aktuellen Orientierung vorrangig für niedergelassene Ärzte, aber auch für onkologisch tätige Spitalsärzte. H. Samonigg, Graz Kompendium Internistische Onkologie Teil II Herausgeber: H. J. Schmoll, K. Höfßcen, K. Possinger (Berlin) Springer, Heidelberg, 1996. 2. Auflage 2172 S., DM 158-ISBN 3-540-60830-3 
Durch Vorlage des zweiten Teils des insgesamt mit knapp 3900 Seiten sehr umfangreichen Kompendiums ist nunmehr die gesamte zweite Auflage verfügbar. Das Werk wendet sich vorrangig an Ärzte, die regelmäßig onkologische Therapien selbst durchführen, aber auch an solche, die nicht an einem onkolo-gischen Zentrum tätig sind, jedoch entsprechende Entscheidungen hinsichtlich Diagnostik und Therapie zu treffen haben. Der zweite Teil des 2bändigen Werkes umfaßt 2172 Seiten und gliedert sich grundsätzlich in zwei Abschnitte: Innerhalb des Kapitels «Therapiekonzepte» werden in äußerst übersichtlicher und umfangreicher Form neben den akuten und chronischen Leukämien, dem Morbus Hodgkin und den Non-HodgkinLymphomen die Tumoren des Kopf-Hals-Be-reichs, des Thorax und des Mediastinums, des Gastrointe-stinaltrakts, der Mamma und der gynäkologischen Tumoren auch die soliden Tumoren im Kindesalter abgehandelt. ledes einzelne Kapitel beinhaltet einleitende Bemerkungen zur Epidemiologie der jeweiligen Krankheitsentität, der Histo-logie, der Stadieneinteilung, der Prognose sowie der Charakte-ristika der Erkrankung und des Krankheitsverlaufs. Der Hauptteil jedes Kapitels ist der Therapiestrategie gewidmet, wobei auf den Stellenwert der Chirurgie, der Strahlentherapie sowie der medikamentösen therapeutischen Maßnahmen jeweils einzeln Bezug genommen wird. Den Abschluß bilden Bemerkungen zur Rezidiv-Salvage-Therapie sowie zur Thera-piekontrolle und Hinweise auf eventuelle laufende klinische Studien und zukünftige Entwicklungen. In übersichtlicher Form werden schließlich die jeweils aktuellen Therapieschemata tabellarisch zusammengefaßt, gefolgt von ausführlichen Literaturhinweisen zur jeweiligen Tumorentität. Es folgt ein knapp 100 Seiten umfassendes Kapitel über spe-zielle Therapiemodalitäten. Hierbei wird einerseits auf den aktuellen Stellenwert regionaler medikamentöser Therapieverfahren (Regionale medikamentöse Therapie von Lebermeta-stasen, intraperitoneale Chemotherapie, intravesikale Therapie, Extremitätenperfusion bei malignen Melanomen etc.) eingegangen, andererseits werden grundsätzhche Therapiestrategien bei malignen Pleuraergüssen und malignen Perikardergüssen sowie die Therapie von Skelettmetastasen und Hirnmetasta-sen und die chirurgische Therapie von Lungenmetastasen ein-gehend beleuchtet.

Hinzuweisen ist weiters auf ein Kapitel über den aktuellen Stand von Chemotherapie und Hyperthermie sowie ein Kapitel über paraneoplastische Syndrome. Den Abschluß bildet ein Beitrag über «Notfallindikationen in der Onkologie». Auf knapp 50 Seiten werden Häufigkeit, Ätiologie sowie Klinik,

\section{KAKGER}

(C) 1997 S. Karger GmbH, Freiburg Fax (07 61) 4520714 http://www.karger.ch

Diagnostik und Therapie sämtlicher relevanter Notfallsitua-tionen in der Onkologie beschrieben. Auch dieses Kapitel ent-hält ausführliche Literaturhinweise.

Im weniger umfangreichen, aber nichtsdestotrotz auch sehr in-formativen 2. Teil des Werkes wird auf die Bewertungskrite-rien der Tumortherapie eingegangen sowie abschließend auf den «Europäischen Kodex gegen Krebs», Der 2. Teil des Kompendiums Internistische Onkologie endet mit einem 141 Seiten umfassenden Fachverzeichnis, welches das enorme Bemühen der Herausgeber widerspiegelt, ein um-fassendes, hochwertiges Nachschlagewerk zur Verfügung zu stellen.

Zusammenfassend handelt es sich bei der 2. Auflage des 2. Bandes des Kompendiums Internistische Onkologie um ein Werk, welches sich bei großem Umfang durch eine vielfältige und höchst übersichtliche Gliederung auszeichnet. Die inhalt-liche Qualität und die Darstellungsweise können als hervor-ragend eingestuft werden. Insgesamt wurde mit dem 2. 
Band nunmehr ein äußerst instruktives Lehrbuch vorgelegt, welches für den onkologisch tätigen Arzt eine sehr wertvolle Informa-tionsquelle darstellt und als Nachschlagewerk zur Basisausstattung in onkologisch tätigen Abteilungen gehören sollte.

H. Samonigg, Graz

\section{«H€OLO€ll}

Fachliteratur, die überzeugt!

ONKOLOGIE

Informiert über kliniknahe Grundlagenforschung, klinische Krebsforschung und -behandlung

Ich abonniere

die Zeitschrift Onkologie

ab Jahrgang

¿ zum regulären Preis von DEM 182.-

a zum Sonderpreis von DEM 91.- für Mitglieder der

a AIIO Ù CAO

a DGHO $\propto$ ÖGHO (ausschließlich an Privatadresse)

Diese Preise gelten für 1997 und verstehen sich inkl. MWSt, e×k1.Versandkosten.

Preisänderungen vorbehalten.

a. Bitte senden Sie mir ein kostenloses Probeheft von Onkologie

Ich kann diese Bestellung innerhalb eißerWoche durch eine einfache Mitteilung an den Verlag wider-Гufen. Das Abonnement gilt zunächst für ein Jahr und verlängert sịch um jeweils ein weiteres Jahr, wenn Ich die Zeitschrift nicht bis I. Dezember des laufenden Jahres abbestelle.

KARGER

\section{S. Karger GmbH}

Postfach

D-79095 Freiburg

(Deutschland)

http:llwww.karger.chl

journalslonklonkdes.htm

Ich bezahle a mit Scheck a auf Rechnung

Bitte belasten Sie diese Bestellung meiner Kreditkarte

a American Express a Eurocard

Karten-Nr, Ablauf

Ich ermächtige die S. Karger GmbH bis aufWiderruf, jährlich meine Abonnement-Gebühren im Lastschriftverfahren abzubuchen (nur innerhalb Deutschlands möglich)

bei Bank oder Post

in

BLZ

Giro-Konto-Nr.

Meine Postanschrift lautet

(Blockschrift) 
Tel.

Datum/Unterschrift 